\title{
Multi-Particle Processes in QCD without Feynman Diagrams
}

\author{
Costas G. Papadopoulos ${ }^{\mathrm{a}}$ and Małgorzata Worek ${ }^{\mathrm{ab} *}$ \\ anstitute of Nuclear Physics, NCSR Demokritos, 15-310 Athens, Greece \\ bInstitute of Nuclear Physics PAS, Radzikowskiego 152, 31-3420, Cracow, Poland
}

A way to efficiently compute helicity amplitudes for arbitrary tree-level scattering processes in QCD is presented. The scattering amplitude is evaluated recursively through a set of Dyson-Schwinger equations. The computational cost of this algorithm grows asymptotically as $3^{n}$, where $n$ is the number of particles involved in the process, compared to $n$ ! in the traditional Feynman graphs approach. Unitary gauge is used and mass effects are available as well. Additionally, the color and helicity structures are appropriately transformed so the usual summation is replaced by the Monte Carlo techniques.

\section{INTRODUCTION}

QCD processes with many external legs are of much interest, both for testing QCD in different settings and as backgrounds for new physics processes at the Fermilab TeVatron and at the CERN LHC. However, the estimation of multi-jet production cross sections as well as their characteristic distributions is a difficult task. Perturbation theory based on Feynman graphs runs into computational problems, since the number of graphs contributing to the amplitude grows like $n$ !. The counting of the graphs itself becomes a problem let alone their evaluation and the computation of the color and helicity structures which is an additional source of computational inefficiencies. Over the last few years, new recursive algorithms based on Dyson-Schwinger equations 123 or on field equations 4567 have been developed in order to overcome the computational obstacles. Very recently also on shell recursive equations have been proposed 89]. The amplitude calculated using Dyson-Schwinger recursive equations, which avoid Feynman diagrams, results in

\footnotetext{
*Presented at the X International Workshop on Advanced Computing and Analysis Techniques in Physics Research, ACAT 2005, DESY-Zeuthen, Germany, 22-27 May 2005.
}

a computational cost growing asymptotically as $3^{n}$. Here, the off-shell subamplitudes are introduced which are combinations of parts of Feynman graphs. For those subamplitudes a recursion relation has been obtained which enables to express an n-particle amplitude in terms of all subamplitudes, starting from $1-, 2-, \ldots$ up to $(n-1)$ particles. The color and helicity structures corresponding to each subamplitude have a simpler form. Moreover, they can be appropriately transformed so the usual summation can be replaced by the Monte Carlo one.

In this article the algorithm based on DysonSchwinger recursive equations is presented and used in order to efficiently obtain cross sections for arbitrary multi-jet processes.

\section{DESCRIPTION OF THE ALGO- RITHM}

To illustrate how the algorithm works let us present a simple example, the gluon selfinteraction:

$g\left(p_{1}\right) g\left(p_{2}\right) \rightarrow g\left(p_{3}\right) g\left(p_{4}\right)$.

Here $p_{1}, p_{2}, p_{3}, p_{4}$ represent the external momenta involved in the scattering process, taken to be in- 
coming. The subamplitude with an off-shell gluon of momentum $P$ has contributions from threeand four-gluon vertices only. To reduce the computational complexity down to an asymptotic $3^{n}$, we replaced the four-gluon vertex with a threegluon vertex by introducing an auxiliary field represented by the antisymmetric tensor $H^{\mu \nu}$. The recursion for the gluons now changes only slightly. However, we have an additional equation for the auxiliary field. Diagrammatically, the full con-

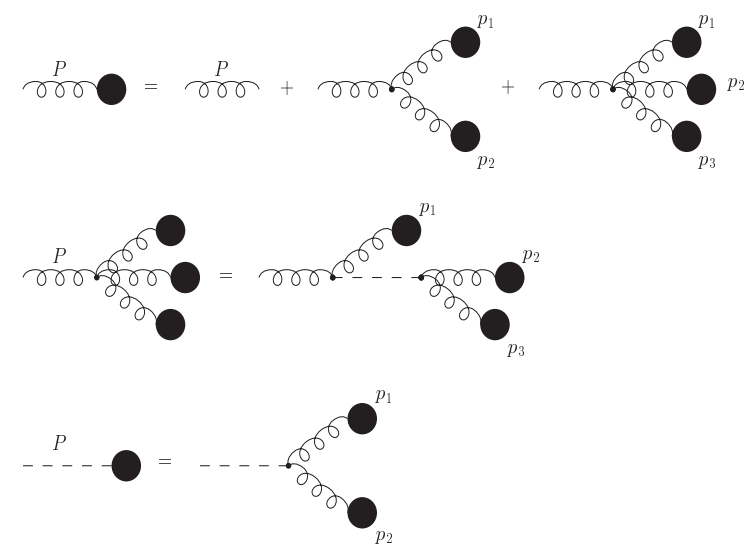

Figure 1. Recursion equations for gluon selfinteractions. The auxiliary field is used to replace the four-gluon vertex in order to reduce computational complexity of calculations.

tent of Dyson-Schwinger equations is presented in Fig. 1 and the corresponding equations have the following form:

$$
\begin{aligned}
& A_{A B}^{\mu}(P)=\frac{g_{s}}{2 P^{2}} \sum_{P=p_{1}+p_{2}} V_{\nu \lambda}^{\mu}\left(P, p_{1}, p_{2}\right) \epsilon\left(p_{1}, p_{2}\right) \\
& \left\{A_{A C}^{\nu}\left(p_{1}\right) A_{C B}^{\lambda}\left(p_{2}\right)-A_{A C}^{\lambda}\left(p_{2}\right) A_{C B}^{\nu}\left(p_{1}\right)\right\} \\
& +\frac{i g_{s}}{2 P^{2}} \sum_{P=p_{1}+p_{2}} X_{\nu \lambda \rho}^{\mu}\left\{A_{A C}^{\nu}\left(p_{1}\right) H_{C B}^{\lambda \rho}\left(p_{2}\right)\right. \\
& \left.-H_{A C}^{\lambda \rho}\left(p_{2}\right) A_{C B}^{\nu}\left(p_{1}\right)\right\} \epsilon\left(p_{1}, p_{2}\right) \\
& H_{A B}^{\mu \nu}(P)=\frac{i g_{s}}{4} \sum_{P=p_{1}+p_{2}} X_{\lambda \rho}^{\mu \nu} \epsilon\left(p_{1}, p_{2}\right)
\end{aligned}
$$

$$
\left\{A_{A C}^{\lambda}\left(p_{1}\right) A_{C B}^{\rho}\left(p_{2}\right)-A_{A C}^{\rho}\left(p_{2}\right) A_{C B}^{\lambda}\left(p_{1}\right)\right\}
$$

where $X^{\mu \nu \lambda \rho}$ is the new auxiliary field-gluongluon vertex:

$X^{\mu \nu \lambda \rho}=g^{\mu \lambda} g^{\nu \rho}-g^{\nu \lambda} g^{\mu \rho}$

and $A, B, C=1,2,3$, and $\epsilon\left(p_{1}, p_{2}\right)$ is the Fermi sign factor. In order to be more transparent we will write explicitly the three-gluon vertex part as well as the auxiliary one, suppressing the color indices and using the light-cone representation:

$$
\begin{aligned}
& A^{\mu}(P) \sim\left(A\left(p_{1}\right) \cdot A\left(p_{2}\right)\right)\left(p_{2}-p_{1}\right)^{\mu}+\left(p_{1} \cdot A\left(p_{2}\right)+\right. \\
& \left.P \cdot A\left(p_{2}\right)\right) A^{\mu}\left(p_{1}\right)-\left(p_{2} \cdot A\left(p_{1}\right)+P \cdot A\left(p_{1}\right)\right) A^{\mu}\left(p_{2}\right) \\
& +A_{\nu}\left(p_{1}\right) H^{\mu \nu}\left(p_{2}\right)-A_{\nu}\left(p_{1}\right) H^{\nu \mu}\left(p_{2}\right) \\
& H^{\mu \nu}(P) \sim A^{\mu}\left(p_{1}\right) A^{\nu}\left(p_{2}\right)-A^{\nu}\left(p_{1}\right) A^{\mu}\left(p_{2}\right)
\end{aligned}
$$

where all momenta are taken to be incoming. These equations, the off-shell fields, are the main building blocks of the gluon self-interaction, which is to be constructed iteratively. After $n-1$ steps, where $n$ is the number of particles under consideration, one can get the total amplitude

$\mathcal{A}\left(p_{1}, p_{2}, \ldots, p_{n}\right)=\hat{A}_{A B}\left(P_{i}\right) \cdot A_{A B}\left(p_{i}\right)$,

where

$P_{i}=\sum_{j \neq i} p_{j}$

so that $P_{i}+p_{i}=0$. The hat denotes functions given by the expression from the previous step except for the propagator term. This is because the outgoing momentum $P_{i}$ must be on shell and the propagator term is removed by the amputation procedure. The iteration begins with the initial condition for the external particles. For gluons we have

$A_{A B}^{\mu}\left(p_{i}\right)=\varepsilon_{\lambda}^{\mu}\left(p_{i}\right) \delta_{A C} \delta_{D B}$,

where $i$ enumerates the external particles, $i=$ $1, \ldots, n, \varepsilon$ denotes the polarization vector and $\lambda=$ \pm 1 .

In order to label and systematically control the momenta of external particles and their relevant intermediate combination, we give all momenta in the binary representation, see e.g. [5]. 
For the process under consideration, and the external particles with momenta $p_{i}^{\mu}, i=1,2,3,4$ we assign to the momentum $P^{\mu}$ a binary vector $\vec{m}=\left(m_{1}, m_{2}, m_{3}, m_{4}\right)$ with components which are either 0 or 1 as follows:

$P^{\mu}=\sum_{i=1}^{4} m_{i} p_{i}^{\mu}$

The binary vector can now be uniquely represented by the integer

$m=\sum_{i=1}^{4} 2^{i-1} m_{i}$.

In particular, one can write:

$g(1) g(2) \rightarrow g(4) g(8)$.

All subamplitudes can now be replaced by the corresponding integers

$A^{\mu}(P) \rightarrow A^{\mu}(m)$,

$H^{\mu \nu}(P) \rightarrow H^{\mu \nu}(m)$.

This representation allows us to establish a natural ordering of the momenta based on the notion of level, defined simply as

$l=\sum_{i=1}^{4} m_{i}$.

All external momenta are at the level 1 , whereas the total amplitude corresponds to the unique level $n=4$. With levels we can see the natural iteration path of the equations. We start from the subamplitudes at the level 1 which are the external momenta together with the initial conditions, then go to the subamplitudes at the level 2 and so on until we reach level $n=4$. The Fermi sign factor is also expressed in the integer number language

$\epsilon\left(P_{1}, P_{2}\right) \rightarrow \epsilon\left(m_{1}, m_{2}\right)$

and we use the following formula

$\epsilon\left(m_{1}, m_{2}\right)=(-1)^{\chi\left(m_{1}, m_{2}\right)}$

with

$\chi\left(m_{1}, m_{2}\right)=\sum_{i=n}^{2} \hat{m}_{1 i} \sum_{j=1}^{i-1} \hat{m}_{2 j}$, where the hat means that this particular component is equal to 0 if the corresponding external particle is a boson. This sign factor takes into account the sign change when two identical fermions are interchanged.

Contrary to orginal HELAC [12], the computational part consists of only one step, where couplings allowed by the lagrangian defined by fusion rules are only explored, see Ref. [10] for technical details. Subsequently, the helicity configurations are set up. There are two possibilities, either exact summation over all $2^{n_{1}} \times 2^{n_{2}} \times 2^{n_{3}}$ helicity configurations, where $n_{1}$ is the total number of quarks, $n_{2}$, the total number of antiquarks and $n_{3}$, the total number of gluons; or Monte Carlo summation. For example for a gluon the second option is achieved by introducing the polarization vector

$\varepsilon_{\phi}^{\mu}(p)=e^{i \phi} \varepsilon_{+}^{\mu}(p)+e^{-i \phi} \varepsilon_{-}^{\mu}(p)$,

where $\phi \in(0,2 \pi)$ is a random number. By integrating over $\phi$ we can obtain the sum over helicities

$\frac{1}{2 \pi} \int_{0}^{2 \pi} d \phi \varepsilon_{\phi}^{\mu}(p)\left(\varepsilon_{\phi}^{\nu}(p)\right)^{*}=\sum_{\lambda= \pm} \varepsilon_{\lambda}^{\mu}(p)\left(\varepsilon_{\lambda}^{\nu}(p)\right)^{*}$.

The same idea can be applied to the helicity of quarks and antiquarks.

Finally, the color factor is evaluated iteratively. Once again, we have two options. Either we proceed by computing all $3^{n_{q}} \times 3^{n_{\bar{q}}}$ color configurations, where the gluon is treated as a quarkantiquark pair and $n_{q}, n_{\bar{q}}$ is the number of quarks and antiquarks respectively, or particular configurations are chosen by the Monte Carlo method (for more details see Ref. [10]).

For the process $g\left(P_{1}\right) g\left(P_{2}\right) \rightarrow g\left(P_{4}\right) g\left(P_{8}\right)$ which we are using through this section as an example the momenta involved in the various levels of calculation are the following:

$\underline{1 \text { st Level: }}$

$P_{1}=(0001)=p_{1}, \quad P_{2}=(0010)=p_{2}$

$P_{4}=(0100)=p_{3}, \quad P_{8}=(1000)=p_{4}$

2nd Level: 
$P_{6}=(0110)=p_{2}+p_{3}$

$P_{10}=(1010)=p_{2}+p_{4}$

$P_{12}=(1100)=p_{3}+p_{4}$

3th Level: $\quad P_{14}=(1110)=p_{2}+p_{3}+p_{4}$

4th Level: $P_{15}=(1111)=p_{1}+p_{2}+p_{3}+p_{4}$.

Note that we have chosen the particle number 1 as our ending point and, therefore, we have only computed all the relevant momentum combinations where the momentum $p_{1}$ does not appear. This excludes all odd integers between 1 and $2^{n}-2$. The momentum $p_{1}$ is combined only once, in the last $n$-th level. Starting from the second level we get:

$$
\begin{aligned}
& A^{\mu}(6) \sim V^{\nu \rho \sigma}(6,2,4) A_{\rho}(2) A_{\sigma}(4) \\
& H^{\mu \nu}(6) \sim X^{\mu \nu \rho \sigma} A_{\rho}(2) A_{\sigma}(4) \\
& A^{\mu}(10) \sim V^{\nu \rho \sigma}(10,2,8) A_{\rho}(2) A_{\sigma}(8) \\
& H^{\mu \nu}(10) \sim X^{\mu \nu \rho \sigma} A_{\rho}(2) A_{\sigma}(8) \\
& A^{\mu}(12) \sim V^{\nu \rho \sigma}(12,4,8) A_{\rho}(4) A_{\sigma}(8) \\
& H^{\mu \nu}(12) \sim X^{\mu \nu \rho \sigma} A_{\rho}(4) A_{\sigma}(8)
\end{aligned}
$$

At level 3:

$$
\begin{aligned}
& A^{\mu}(14) \sim V^{\mu \rho \sigma}(14,2,12) A_{\rho}(2) A_{\sigma}(12)- \\
& X^{\mu \nu \rho \sigma} A_{\nu}(2) H_{\rho \sigma}(12)+V^{\mu \rho \sigma}(14,4,10) A_{\rho}(4) A_{\sigma}(10) \\
& -X^{\mu \nu \rho \sigma} A_{\nu}(4) H_{\rho \sigma}(10)+V^{\mu \rho \sigma}(14,8,6) A_{\rho}(8) A_{\sigma}(6) \\
& -X^{\mu \nu \rho \sigma} A_{\nu}(8) H_{\rho \sigma}(6) .
\end{aligned}
$$

At the last, 4-th level, the total amplitude is computed by combining this subamplitude with the remaining one, which describes the momentum $p_{1}$ and is simply given by

$\mathcal{A}(15) \sim A_{\mu}(1) \cdot A^{\mu}(14)$.
Table 1

Results for the total cross section from 4 up to 8 gluons, $\sigma_{\mathrm{T}}$ corresponds to summation over all possible color configurations, while $\sigma_{\mathrm{T}}^{\mathrm{MC}}$ corresponds to Monte Carlo summation.

\begin{tabular}{||l|l|l||}
\hline \hline Process & $\sigma_{\mathrm{T}} \pm$ error $(\mathrm{nb})$ & $\sigma_{\mathrm{T}}^{\mathrm{MC}} \pm$ error $(\mathrm{nb})$ \\
\hline \hline$g g \rightarrow 2 g$ & $4611.55 \pm 38.13$ & $4627.18 \pm 33.28$ \\
\hline$g g \rightarrow 3 g$ & $152.444 \pm 2.490$ & $152.137 \pm 2.822$ \\
\hline$g g \rightarrow 4 g$ & $12.9072 \pm 0.4070$ & $12.6137 \pm 0.4619$ \\
\hline$g g \rightarrow 5 g$ & $1.04254 \pm 0.05300$ & $1.04446 \pm 0.10390$ \\
\hline$g g \rightarrow 6 g$ & $0.07577 \pm 0.00597$ & $0.07261 \pm 0.00516$ \\
\hline \hline
\end{tabular}

\section{NUMERICAL RESULTS}

As an example the algorithm has been used to compute total cross sections for multiple jets production. The CMS energy was chosen $\sqrt{s}=14$ $\mathrm{TeV}$ and the following cuts were applied

$p_{T_{i}}>60 \mathrm{GeV}, \quad\left|\eta_{i}\right|<2.5, \quad \Delta R>1.0$

where $p_{T}=\sqrt{p_{x}^{2}+p_{y}^{2}}$ is the transverse momentum of a jet, $\eta=-\ln \tan (\theta / 2)$ is the pseudorapidity. Many methods can be used to define what is meant by a jet of hadron. One commonly used is the 'cone' description of a jet which is the transverse energy, $E_{T}$, concentration in a cone of radius

$\Delta R=\sqrt{\Delta \Phi_{i}^{2}+\Delta \eta_{i j}^{2}}$

with

$\Delta \Phi_{i j}=\arccos \left(\frac{p_{x_{i}} p_{x_{j}}+p_{y_{i}} p_{y_{j}}}{p_{T_{i}} p_{T_{j}}}\right)$.

All results are obtained with a fixed strong coupling constant calculated at the $M_{Z}$ scale. There are several parameterizations for the parton structure functions, we used CTEQ6 PDF's parametrization [1112. For the phase space generation we used either PHEGAS [13] or a flat phasespace generator RAMBO [14].

The results for the total cross section from 4 up to 8 gluons are listed above in Table 1 We give 

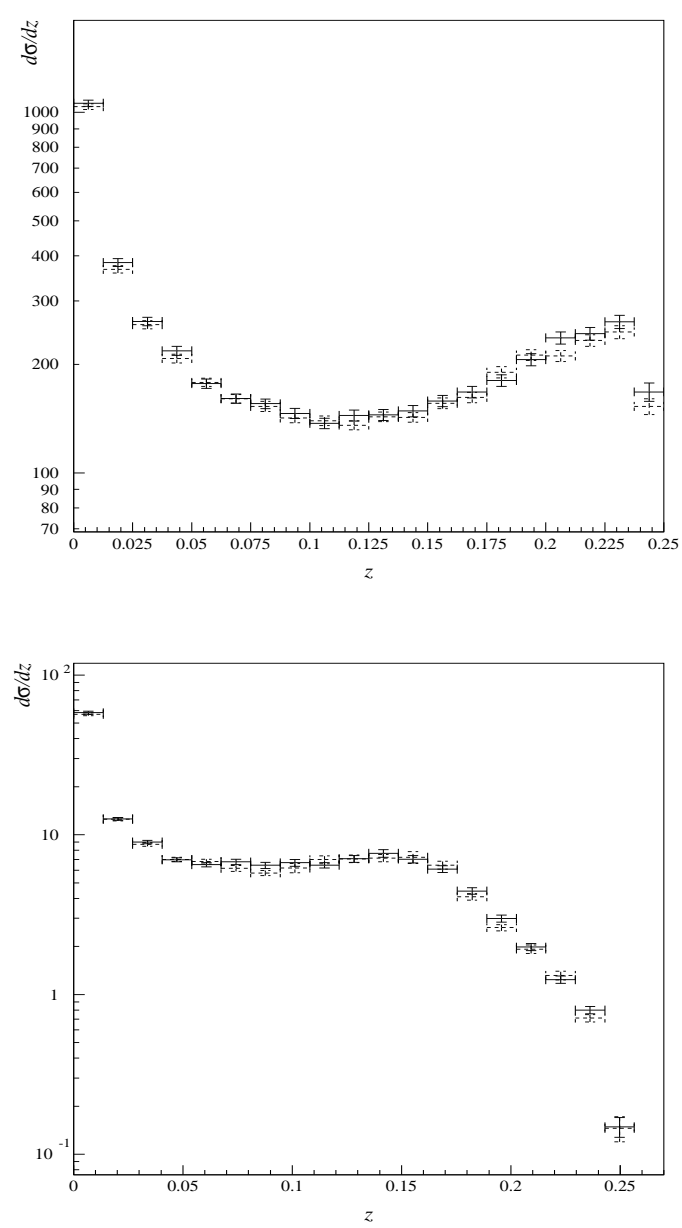

Figure 2. Distribution in $z=\left|M_{I}\right|^{2} / \sum_{i}^{\text {all }}\left|M_{i}\right|^{2}$, where $\left|M_{I}\right|^{2}$ is the square matrix element for one particular color configuration normalized to the sum of all possible is plotted. The upper plot corresponds to the $g g \rightarrow g g$ process, the lower one to the $g g \rightarrow$ ggg. Solid line crosses denote summation over all color configurations whereas dashed, the Monte Carlo summation.

the 'exact' result with summation over all possible color configurations $\left(10^{6}\right.$ generated events $)$, $\sigma_{\mathrm{T}}$ as well as the result obtained with Monte Carlo summation over color $\left(4 \times 10^{6}\right.$ generated events $)$ $\sigma_{\mathrm{T}}^{\mathrm{MC}}$. In both cases a Monte Carlo over helicity is applied.

The next result we present is an example of summation over all color configurations in comparison to Monte Carlo summation. In Fig. 22 the $\mathrm{X}$-axis variable is

$$
z=\left|M_{I}\right|^{2} / \sum_{i}^{\text {all }}\left|M_{i}\right|^{2}
$$

where $\left|M_{I}\right|^{2}$ is the square matrix element for one particular color configuration normalized to the sum of all possible is plotted. The agreement is easy visible.

\section{SUMMARY}

An efficient tool for automatic computation of helicity amplitudes and cross sections for multiparticle final states in QCD has been presented.

\section{Acknowledgments}

Work supported by the Polish State Committee for Scientific Research Grants number 1 P03B 00927 for years 2004-2005 (M.W.). In addition, M.W. acknowledges the Maria Curie Fellowship granted by the the European Community in the framework of the Human Potential Programme under contract HPMD-CT-2001-00105 ("Multiparticle production and higher order correction").

\section{REFERENCES}

1. A. Kanaki and C. G. Papadopoulos, Comput. Phys. Commun. 132 (2000) 306-315, hep-ph/0002082

2. A. Kanaki and C. G. Papadopoulos, hep-ph/0012004

3. P. D. Draggiotis, R. H. P. Kleiss, and C. G. Papadopoulos, Eur. Phys. J. C24 (2002) 447-458, hep-ph/0202201

4. F. A. Berends and W. Giele, Nucl. Phys. B294 (1987) 700.

5. F. Caravaglios and M. Moretti, Phys. Lett. B358 (1995) 332-338, hep-ph/9507237

6. P. Draggiotis, R. H. P. Kleiss, and C. G. Papadopoulos, Phys. Lett. B439 (1998) 157164, hep-ph/9807207. 
7. F. Caravaglios, M. L. Mangano, M. Moretti, and R. Pittau, Nucl. Phys. B539 (1999) 215232, hep-ph/9807570

8. R. Britto, F. Cachazo, and B. Feng, Nucl. Phys. B715 (2005) 499-522, hep-th/0412308

9. R. Britto, F. Cachazo, B. Feng, and E. Witten, Phys. Rev. Lett. 94 (2005) 181602, hep-th/0501052

10. C.G. Papadopoulos and M. Worek, in preparation.

11. J. Pumplin et al., JHEP 07 (2002) 012, hep-ph/0201195

12. D. Stump et al., JHEP 10 (2003) 046, hep-ph/0303013

13. C. G. Papadopoulos, Comput. Phys. Commun. 137 (2001) 247-254, hep-ph/0007335

14. R. Kleiss, W. J. Stirling, and S. D. Ellis, Comput. Phys. Commun. 40 (1986) 359. 